\title{
PHOTOLUMINESCENCE OF nc-Si:Er THIN FILMS OBTAINED BY PHYSICAL AND CHEMICAL VAPOUR DEPOSITION TECHNIQUES: THE EFFECTS OF MICROSTRUCTURE AND CHEMICAL COMPOSITION
}

\author{
M.F. Cerqueira, ${ }^{1, a}$, M. Losurdo ${ }^{2}$, M. Stepikhova ${ }^{3}$, P. Alpuim ${ }^{1}$, G. Andres ${ }^{1}$, A. \\ Kozanecki ${ }^{4}$, M.J. Soares ${ }^{5}$, M. Peres ${ }^{5}$ \\ ${ }^{1}$ Departamento de Física, Universidade do Minho, Campus de Gualtar 4710-057 Braga, Portugal \\ ${ }^{2}$ Institute of Inorganic Methodologies and of Plasmas, IMIP-CNR, Via Orabona n.4-70126 Bari, Italy \\ ${ }^{3}$ Institute for Physics of Microstructures RAS, 603600 Nizhnij Novgorod GSP-105, Russia \\ ${ }^{4}$ Polish Academy of Sciences, Institute of Physics, PL-02668, Warsaw, Poland \\ ${ }^{5}$ Departamento de Física, Universidade de Aveiro, Campus de Santiago, 3700 Aveiro, Portugal \\ afcerqueira@fisica.uminho.pt
}

Keywords: Erbium, nanocrystalline silicon, photoluminescence

\begin{abstract}
Erbium doped nanocrystalline silicon (nc-Si:Er) thin films were produced by reactive magnetron rf sputtering and by Er ion implantation into chemical vapor deposited Si films. The structure and chemical composition of films obtained by the two approaches were studied by microRaman scattering, spectroscopic ellipsometry and Rutherford backscattering techniques. Variation of deposition parameters was used to deposit films with different crystalline fraction and crystallite size. Photoluminescence measurements revealed a correlation between film microstructure and the $\mathrm{Er}^{3+}$ photoluminescence efficiency.
\end{abstract}

\section{Introduction}

Since the discovery of room temperature (RT) photoluminescence (PL) in the visible range from porous silicon, research efforts on nanostructured silicon-based materials have been aimed at realizing efficient Si-based light emitters. Among the various structures and approaches, erbium (Er) doped nanocrystalline silicon, nc-Si, has received great attention in the last ten years [1-3], since evidence was reported that nc-Si:Er yields enhanced intra-ionic luminescence and reduced PL thermal quenching when compared to crystalline $\mathrm{Si}$ [2, 4-6]. However, controversial results have been obtained for the Er-related radiative lifetime and quenching mechanisms, as pointed out in ref [7]. Most of the previous studies were focused on $\mathrm{Si}$ nanocrystals embedded in $\mathrm{SiO}_{2}$, which is transparent in the 
visible-IR range. Few studies have been reported on the $\mathrm{Er}^{3+} \mathrm{PL}$ properties for material containing $\mathrm{Si}$ nanocrystals embedded in an amorphous silicon matrix with or without oxygen and hydrogen [8]. This is the purpose of the present work.

In this paper, we discuss the dependence of the $\mathrm{Er}^{3+} \mathrm{PL}$ on the silicon film microstructure (i.e., crystalline fraction and crystallite size) and chemical composition (i.e., hydrogen and oxygen content in the silicon network). The erbium doping of the thin films was obtained using two different approaches: i) in-situ doping for the sputtered samples, where the crystalline Si target was covered with pieces of metallic Er, and ii) Er-implantation for the silicon layers grown by chemical vapour deposition, CVD (both hot wire CVD and plasma enhanced CVD).

\section{Experimental details}

The in-situ doped nc-Si:Er thin films were grown in an $\mathrm{Ar} / \mathrm{H}_{2}$ atmosphere by radiofrequency (rf) magnetron sputtering (physical vapour deposition-PVD) of c-Si target with small pieces of metallic erbium added outside the erosion area. Ordinary glass plates were used as substrates. Samples with different silicon crystal size and crystalline volume fraction (microstructure) and oxygen and hydrogen contents were obtained by changing the rf power and the substrate temperature. More details concerning the deposition by sputtering can be found in ref. [9].

Another set of samples was deposited by Hot Wire CVD (HWCVD) and Plasma Enhanced CVD (rfPECVD) in a ultra high vacuum system with a base pressure $\leq 2.7 \times 10^{-5} \mathrm{~Pa}$ on double-polished crystalline silicon (c-Si) wafers. For the HW-CVD grown samples, a single tungsten filament of 0.5 $\mathrm{mm}$ in diameter and approximately $14 \mathrm{~cm}$ in length was bent to a coil and placed $4 \mathrm{~cm}$ from the substrate. It was resistively heated with an AC power supply. The filament temperature of $1950{ }^{\circ} \mathrm{C}$ was measured by a two-colour digital ratio pyrometer and the pressure during deposition was kept constant at 2.7 Pa. For the rf-PECVD grown samples, the inter-electrode distance was $3 \mathrm{~cm}$, the density of the rf power was $175 \mathrm{~mW} / \mathrm{cm}^{2}$ and the pressure was $20 \mathrm{~Pa}$. For both methods, the silane flux, $\Phi_{\mathrm{SiH} 4}$, was kept at $1 \mathrm{sccm}$ and the hydrogen flux, $\Phi_{\mathrm{H} 2}$, was varied in order to obtain the desired dilution, defined as $\mathrm{D}_{\mathrm{H}}$ $=\Phi_{\mathrm{H} 2} /\left(\Phi_{\mathrm{H} 2}+\Phi_{\mathrm{SiH} 4}\right)$.

Hydrogenated amorphous silicon (a-Si:H) films were obtained at $0 \% \mathrm{H}_{2}$ dilution by $\mathrm{HW}$ and rfPECVD, while highly crystalline films (nc-Si:H) were deposited at $96 \% \mathrm{H}_{2}$ dilution. After deposition, films were implanted with erbium using a beam flux of $1 \times 10^{14} \mathrm{~atm} / \mathrm{cm}^{2}$. Annealing treatments at 375 ${ }^{\circ} \mathrm{C}$ for $30 \mathrm{~min}$ in a nitrogen atmosphere were performed to recover from the implantation damage. Thermal annealing can cause different structural and chemical modifications of the samples, including 
recovering from the implantation damage, atomic diffusion (modification of the $\mathrm{O}$ and $\mathrm{H}$ profiles), atomic rearrangement and crystallization [10-12]. The deposition conditions of all the samples are summarized in Table 1.

Table 1 - Growth conditions for Erbium doped nanocrystalline silicon thin films. $D_{\mathrm{H}}=\Phi_{\mathrm{H} 2} /\left(\Phi_{\mathrm{H} 2}+\Phi_{\mathrm{SiH} 4}\right)$ is the hydrogen dilution used for CVD deposition ( $\Phi_{\mathrm{H} 2}$ and $\Phi_{\mathrm{SiH} 4}$ are the flow rates of $\mathrm{H}_{2}$ and $\left.\mathrm{SiH}_{4}\right) . \mathrm{R}_{\mathrm{H}}=\mathrm{p}_{\mathrm{H} 2} /\left(\mathrm{p}_{\mathrm{H} 2}+\mathrm{p}_{\mathrm{Ar}}\right)$

\begin{tabular}{|c|c|c|c|c|}
\multicolumn{5}{|c|}{0.63 was the hydrogen fraction for all the sputtered samples. } \\
\hline Sample & Method & $\begin{array}{c}\text { Temperature } \\
\left({ }^{\circ} \mathrm{C}\right)\end{array}$ & rf power $(\mathrm{W})$ & $\begin{array}{c}\mathrm{D}_{\mathrm{H}} \\
(\%)\end{array}$ \\
\hline Er34 & Sputtering & 50 & 80 & - \\
\hline P13 & Sputtering & 150 & 80 & - \\
\hline P14 & Sputtering & 200 & 80 & - \\
\hline P15 & Sputtering & 300 & 40 & - \\
\hline P28 & Sputtering & 150 & 40 & - \\
\hline Si35 & HW/CVD & 220 & - & 96 \\
\hline Si36 & HW/CVD & 220 & - & 0 \\
\hline Si37 & rf/PECVD & 240 & 5 & 0 \\
\hline
\end{tabular}

The chemical composition was studied by combining the Rutherford Backscattering Spectroscopy (RBS) and Elastic Recoil Detection techniques. The nanocrystal (NC) size and relative fraction of the crystalline phase were estimated using the standard procedure based on Raman spectra, transmission electron microscopy and X-ray diffraction studies as described in ref [13].

Spectroscopic ellipsometry (SE) was applied in order to assess an effective dielectric function of the films and through it, to analyze the film microstructure and chemical composition. SE spectra of the real, $\left\langle\varepsilon_{1}\right\rangle$, and imaginary, $\left\langle\varepsilon_{2}\right\rangle$, parts of the complex pseudo dielectric function were measured in the $0.75-5.5 \mathrm{eV}$ energy range using a phase modulated spectroscopic ellipsometer (UVISEL- Jobin Yvon) at an angle of incidence of $70^{\circ}$. The spectra were analyzed using models based on the Bruggeman effective medium approximation [14] according to the approach reported in ref. [15]. This allowed us to estimate the matrix chemical composition, the Er content and the films crystalline volume fraction.

Photoluminescence measurements in the infrared spectral region were performed with a SPEX75 monochromator. The signal was detected by a germanium detector using the $514.5 \mathrm{~nm}$ line of an $\mathrm{Ar}^{+}$ laser (laser power on the sample was $150 \mathrm{~mW}$ ) for the excitation. The Er-related PL was studied in the temperature range of $6-300 \mathrm{~K}$. 


\section{Results and discussion}

Table 2 summarizes the Er-content and the $\mathrm{Si}: \mathrm{O}: \mathrm{H}$ ratio for the various samples (oxygen was present at a contamination level during the deposition procedure). Films deposited by CVD are characterized by low oxygen content whereas the sputtered has resulted in larger oxygen content.

Figure 1 shows the Raman spectra of films deposited by sputtering (a) and CVD (b). The presence of silicon nanocrystals are seen by the appearance of the TO mode characteristic of c-Si (approximately $520 \mathrm{~cm}^{-1}$ ). For films deposited by sputtering the crystallinity increases with decreasing the rf power and for a fixed rf power the crystallinity increases with the substrate temperature. For films deposited by CVD, the microstructure is amorphous for films grown without hydrogen dilution, otherwise the microstructure is microcrystalline for $96 \% \mathrm{H}_{2}$ dilution.

The Er34 sample, has been extensively characterized by transmission electron microscopy (see ref [8]) showing a volume fraction of $34 \%$ of silicon nanocrystallites with a size $<3 \mathrm{~nm}$ embedded in an a-Si:H matrix. Otherwise, Si36 and Si37 samples, have not silicon crystals. These samples are purely hydrogenated amorphous silicon.

Then, by PVD and CVD we produced three groups of erbium doped silicon samples: (i) one group of samples having Si crystal sizes lower than $3 \mathrm{~nm}$ (Er34, P13 and P14), (ii) one group of hydrogenated amorphous silicon (Si36, Si37) and (iii) other group of bigger crystals size silicon samples (Si35, P15 and P28). These conclusions based on the X-ray diffraction data correlates with the shape and peak position of their Raman spectra (see Fig 1). The estimated values, based on Raman spectra, of the crystalline volume fraction, $C_{R}$, and of the crystallite size, $D_{R}$, are given in Table 2.

Table 2 - Atomic concentration and structural parameters: $D_{R}$ - average crystal size by Raman; $C_{R}$ - crystalline volume fraction by Raman.

\begin{tabular}{|c|c|c|c|c|c|c|}
\hline Sample & $\begin{array}{c}\mathrm{Er} \\
\text { (at\%) }\end{array}$ & $\begin{array}{c}\mathrm{Si} \\
\text { (at\%) }\end{array}$ & $\begin{array}{c}\mathrm{O} \\
\text { (at\%) }\end{array}$ & $\begin{array}{c}\mathrm{H} \\
\text { (at\%) }\end{array}$ & $\begin{array}{c}\mathrm{D}_{\mathrm{R}} \\
(\mathrm{nm})\end{array}$ & $\begin{array}{c}\mathrm{C}_{\mathrm{R}} \\
(\%)\end{array}$ \\
\hline $\mathrm{Er} 34$ & 0.03 & 72 & $<4$ & 27.5 & $<3$ & $<34$ \\
\hline $\mathrm{P} 13$ & 0.55 & 70 & 9 & 17.7 & $<3$ & $<34$ \\
\hline $\mathrm{P} 14$ & 1.4 & 74 & 14 & 9 & $<3$ & $<34$ \\
\hline $\mathrm{P} 15$ & 0.6 & 65 & 10 & 24.9 & 7 & 52 \\
\hline $\mathrm{P} 28$ & 1.8 & 71 & 15 & 11 & 7.2 & 41 \\
\hline $\mathrm{Si35}$ & 0.04 & $\ldots$ & $<4$ & 8 & $>8$ & 63 \\
\hline $\mathrm{Si} 36$ & 1.3 & $\ldots$ & $<4$ & 17 & - & 0 \\
\hline $\mathrm{Si37}$ & 1.3 & $\ldots$ & $<4$ & 20 & - & 0 \\
\hline
\end{tabular}


A deeper insight into film nanostructure has been obtained by the spectroscopic ellipsometry. The ellipsometric analysis for the samples P13 and P14 (with O content >9 at.\% by RBS) indicates an oxygen rich matrix (a-Si:H:O) with a Tauc band gap of $1.96 \pm 0.01 \mathrm{eV}$. The dielectric function derived for these a-Si:H:O matrix is compared to that of high density a-Si from CVD ("standard" sample) in Fig 2. It shows a shift to higher energy of the fundamental absorption edge and a decrease of the amplitude of $\left\langle\varepsilon_{2}\right\rangle$ for our P13 and P14 samples, compared to a "standard" a-Si sample (ref [16]). These effects can be due to an inhomogeneous distribution of oxygen and hydrogen.
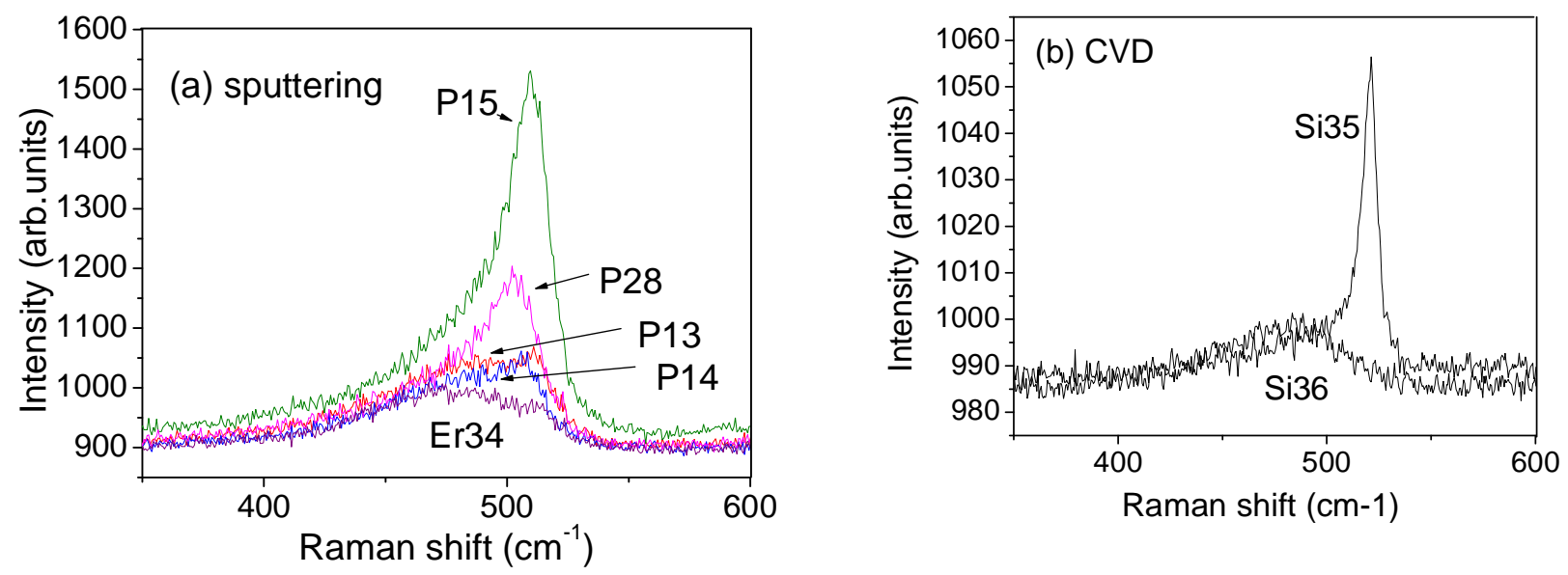

Figure 1 - Raman spectra of films obtained by: (a) Er co-sputtering and (b) HW- and rf-PECVD followed by Er implantation.

By ellipsometry, for each group of samples, the main difference between CVD and the sputtered samples is the presence in the first one of an amorphous surface layer, probably originated by the damage by the Er-implantation, which is not fully recovered by annealing.

With the aim of investigating how these various microstructures (crystal size, crystalline volume fraction and chemical matrix composition) influence the $\mathrm{Er}^{3+}$ photoluminescence, the room temperature PL spectra of the studied samples are compared in Fig. 3. All samples exhibit a characteristic band with a maximum around $1.535 \mu \mathrm{m}$, which is attributed to the $\mathrm{Er}^{3+}$ ions. Interestingly, the most intense $\mathrm{Er}^{3+} \mathrm{PL}$ peak is recorded for the sample with the lowest erbium content $\left(\mathrm{Er}^{3+} \approx 0.03\right.$ at.\%). Furthermore, the $\mathrm{Er}^{3+} \mathrm{PL}$ increases mainly with the decrease of crystallite size, since 
the $\mathrm{Er}^{3+} \mathrm{PL}$ is more intense for the samples having small sized Si nanocrystals $(<3 \mathrm{~nm})$, than for the larger sized ones (with average crystal size $>7 \mathrm{~nm}$ ). From this fact we can infer that the energy transfer between nanocrystals and $\mathrm{Er}^{3+}$ ions is more efficient for small nanocrystals. Furthermore the $\mathrm{Er}^{3+} \mathrm{PL}$ of Si35 (having very low PL intensity-not shown) and Er34 samples, having approximately the same Er content, confirms this effect of small sized crystals on the Er excitation efficiency.



Figure 2 - Spectra of the real, $\varepsilon_{1}$, and imaginary part, $\varepsilon_{2}$, of the dielectric function of the a-Si:H:O matrix characterizing the Er-doped films P13 and P14 obtained by Er co-sputtering. For comparison the dielectric function of a-Si from ref. [16] is also shown.

Interestingly is also to note that the samples having larger size nanocrystals (Si35, P15 and P28) shows lower $\mathrm{Er}^{3+} \mathrm{PL}$ than the purely amorphous ones (Si36 and Si37).

Since from the chemical point of view, the oxygen content for Si36 and Si37 (without Si nanocrystals) is the same as for Er34 (with Si nanocrystals size $<3 \mathrm{~nm}$ ), and also the hydrogen content is high in the three samples, we infer that the $\mathrm{Er}^{3+} \mathrm{PL}$ intensity is mainly affected by the nanocrystal size, rather than the $\mathrm{Si}-\mathrm{O}$ or $\mathrm{Si}-\mathrm{H}$ environment. Then we can say that the presence of small Si nanocrystals favours the energy transfer to the erbium ions.

If we now analyse the $\mathrm{Er}^{3+} \mathrm{PL}$ of the samples with nanocrystals size $<3 \mathrm{~nm}$ it can inferred that, at room temperature, the $\mathrm{Er}^{3+} \mathrm{PL}$ is more efficient for samples containing the small size nanocrystallites embedded in an a-Si:H matrix (Er34 sample), than when they are embedded in an oxygen rich amorphous matrix (a-Si:H:O for P13 and P14 samples), according with the SE results. 

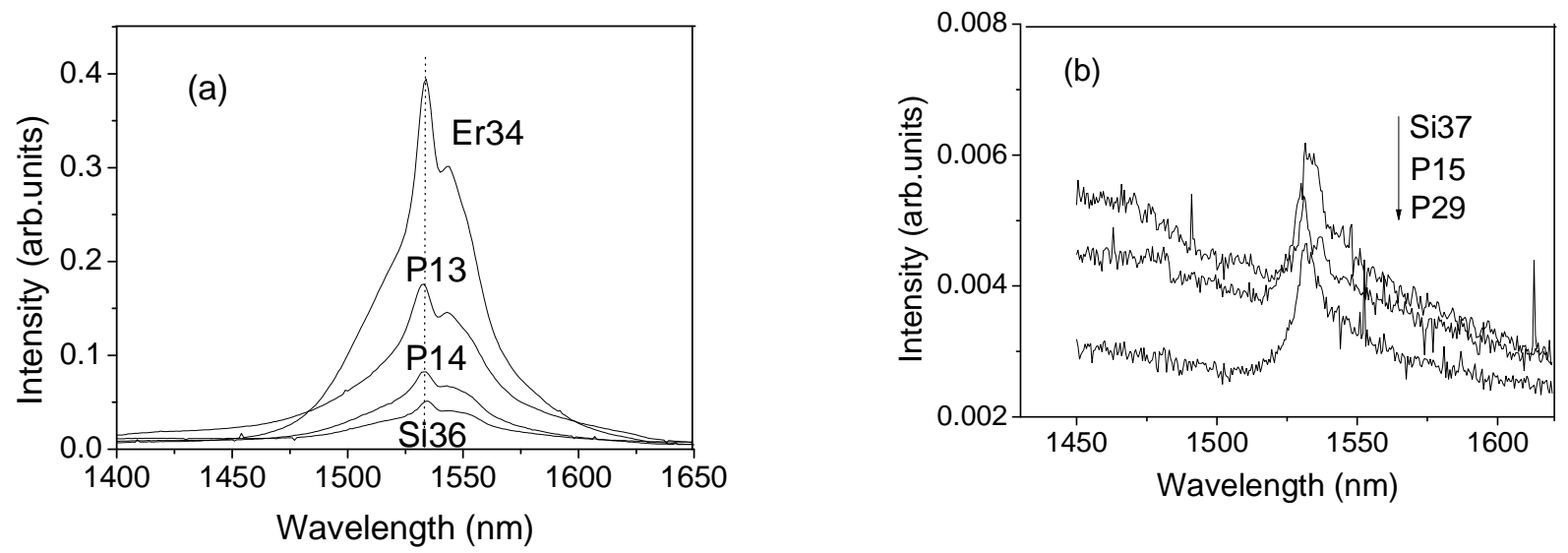

Figure 3 - Infra Red photoluminescence spectra at room temperature for the studied samples, obtained under $514.5 \mathrm{~nm}$ laser line excitation. (a)- Samples with low silicon crystalline fraction; (b) - samples with high crystalline fraction. The implanted samples have been annealed at $375{ }^{\circ} \mathrm{C}$.

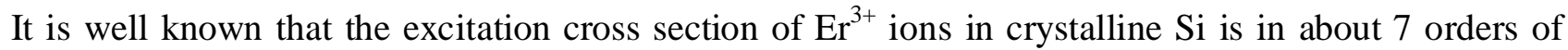
magnitude higher, than in corresponding amorphous materials [17]. Therefore an increase of the $\mathrm{Er}^{3+}$ PL intensity should be expected with the presence of nanocrystals (as observed). On the other hand, the sensitization effect of $\mathrm{Er}^{3+}$ by nanocrystals is less pronounced for larger crystallites (as observed) because the energy gap lowers with the increase of crystallite size and will not match to the $\mathrm{Er}^{3+}$ intracenter transitions.

It should be mentioned that the $\mathrm{Er}^{3+} \mathrm{PL}$ peak for the implanted samples (Si35, Si36, Si37) only appears after the annealing treatment at $300^{\circ} \mathrm{C}$. The observed_PL increase after the annealing could be due to either the formation of Si nanocrystals, or disappearance of some structural defects, or exodiffusion of hydrogen or oxygen occurring during the process. For the annealing temperature used, and since the electrical conductivity of these samples remains (before and after annealing) with typical values of amorphous silicon, the most important effect is probably the decrease of the number of defects, and consequently, the reduction of non-radiative pathways.

Figure 4a demonstrates low-temperature $\mathrm{Er}^{3+} \mathrm{PL}$ spectra, while Fig. $4 \mathrm{~b}$ shows the $\mathrm{Er}^{3+} \mathrm{PL}$ maximum peak intensity as a function of temperature. It should be noted that the sample Si36 shows at low temperature (Fig 4a) the most intense $\mathrm{Er}^{3+} \mathrm{PL}$ signal, in contrast to RT spectra (Fig 3a). Since this sample is purely amorphous (without silicon nanocrystals) then the erbium ions are efficiently excited by the amorphous matrix. Furthermore also confirms the presence and role of non-radiative pathways 
in the decrease of the $\mathrm{Er}^{3+} \mathrm{PL}$ with the increase of temperature.

The temperature dependence of the PL intensity shows two distinct quenching processes and was analyzed by the following fit function, assuming a characteristic thermally deactivated excitation process, according to [18]:

$$
I(T)=I(0)\left[1+C_{1} \exp \left(-A_{1} / k T\right)+C_{2} \exp \left(-A_{2} / k T\right)\right]^{-1},
$$

where $A_{1}$ is the deactivation energy for the high temperature range and $A_{2}$ is the corresponding energy for the low temperature region. The coefficients $\mathrm{C}_{1}$ and $\mathrm{C}_{2}$ are the coupling constants and take into account the excitation/deactivation processes of Er ions. The fitting parameters used to model temperature-dependent $\mathrm{Er}^{3+}$-PL behavior are presented in Table 3, as also the ratio ( $\mathrm{R}$ ) between RT PL and 10K PL.

Table 3 - Values of the curve-fitting parameters used to model temperature-dependent $\mathrm{Er}^{3+}$-PL behavior with eq. (1)

\begin{tabular}{cccccc} 
Sample & $C_{1}$ & $A_{1}(\mathrm{meV})$ & $C_{2}$ & $A_{2}(\mathrm{meV})$ & $R^{*}$ \\
\hline Er34 & 20 & 91 & 0.84 & 8 & 0.42 \\
P13 & 800 & 125 & 1.42 & 6 & 0.10 \\
P14 & 300 & 108 & 1.36 & 5 & 0.13 \\
P15 & 48.8 & 127 & 0.72 & 6 & 0.50 \\
P28 & 28 & 45 & 1.36 & 6 & 0.16 \\
Si35 & 32000 & 157 & 2.9 & 16 & 0.007 \\
Si36 & 18000 & 153 & 2.26 & 8 & 0.017 \\
Si37 & 1400 & 170 & 1.14 & 10 & 0.037 \\
\hline$* R=I_{P_{L}} @ R T / I_{p_{L}} @ 10 K$ & & &
\end{tabular}

Different PL behaviour with the change of temperature is observed as can be seen in Table 3. It is visible that both the thermalization energies and the coupling constants are bigger for the $\underline{C V D}$ samples compared with the sputtered ones. The low temperature erbium thermalization energy is around $6 \mathrm{meV}$ (between 5 and $8 \mathrm{meV}$ ) for the sputtered samples and higher than $8 \mathrm{meV}$ for the $\underline{\mathrm{CVD}}$ ones. The high temperature erbium thermalization energy is around $100 \mathrm{meV}$ for the sputtered samples and higher than $150 \mathrm{meV}$ for the CVD ones, as shown in Table 3. 

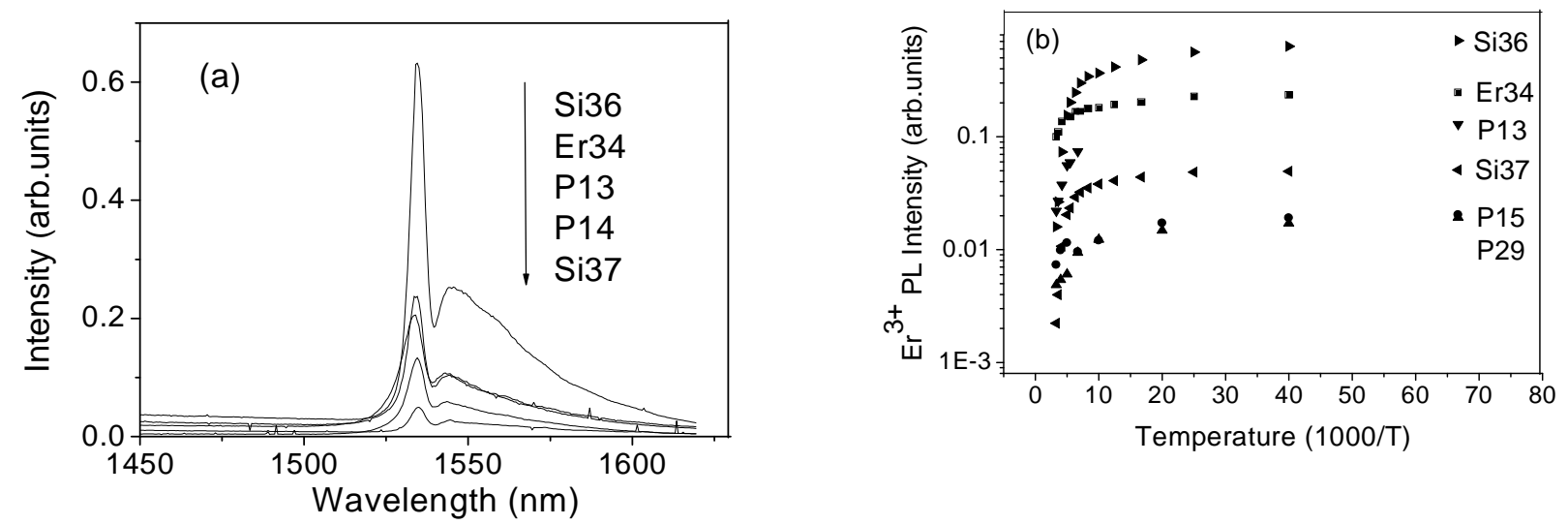

Figure 4 - (a) Infra Red photoluminescence spectra at $20 \mathrm{~K}$ for the studied samples. (b) $\mathrm{Er}^{3+}$ PL intensity as a function of temperature for the studied samples.

Although the big difference, in the thermalization energy, between the low and high temperature ranges, according to the coupling constants values, the de-excitation process responsible for the decrease of the Er emission in our samples seems to be the high thermalization energy component, independently of the growth method used [18].

Furthermore, comparing the $\mathrm{Er}^{3+} \mathrm{PL}$ intensity (Fig 4a) and the ratio ( R ) between RT PL and 10K PL, it is visible that although the samples purely amorphous (Si36 and Si37) or with small nanocrystals (Er34, P13, P14) present a higher efficiency for erbium excitation than the more crystalline ones (P28, P15 and Si35), the PL quenching cannot be directly related with crystallinity, as can be seen in Table 3. In particular, it is seen that this ratio is less than $4 \%$ for the CVD samples, independently either of microstrucutre (amorphous or nanocrystalline silicon) and hydrogen content, and higher than $10 \%$ for the sputtered samples, reaching 42\% for Er34 (Si crystal size $<3 \mathrm{~nm}$ ) and 50\% P15 (Si crystal size of 7 $\mathrm{nm})$. Therefore, from these results we can infer that the $\mathrm{Er}^{3+} \mathrm{PL}$ is more temperature stable for the samples synthesized by Er co-sputtering than for the Er-implantation. This can be due to the residual implantation damage still present in the CVD films even after the annealing treatment at $375^{\circ} \mathrm{C}$.

The highest ratio, R, was obtained for sputtered samples with high hydrogen content (low crystal size Er34 or high crystal size - P15), then we can conclude that hydrogen plays an important role in the PL behaviour with temperature. The beneficial role of hydrogen in improving optical and electrical quality of amorphous silicon films is well known due to passivation of silicon dangling bonds (DB's). For these samples having silicon nanocrystals, the defects, in particular the DB`s, are located in the amorphous matrix, where the NC's are embedded, and at the grain boundaries. The role of atomic 
hydrogen in these films is to saturate both types of DB's and to passivate other defects, therefore reducing the presence of non-radiative recombination pathways.

Therefore, from these results we can infer that, although the more crystalline samples present a less efficient erbium excitation (Fig 3), the PL quenching magnitude can not be related with the size of the Si crystals, but seems to be related with the presence of hydrogen.

\section{Summary and conclusions}

In summary, Er-doped nanocrystalline silicon thin films have been synthesized by reactive magnetron rf sputtering and by implantation of HW and rf-PECVD silicon films. Films with different crystalline fraction and crystal size (ranging from $<3 \mathrm{~nm}$ to $>8 \mathrm{~nm}$ ) and with various oxygen and hydrogen contents have been obtained by changing the deposition parameters. The influence of silicon crystal size and matrix chemical composition on the $\mathrm{Er}^{3+} \mathrm{PL}$ efficiency has been investigated. In particular,

ours results show the following: (i) the $\mathrm{Er}^{3+} \mathrm{PL}$ peak is more intense for the sample with the lowest erbium content $\left(\mathrm{Er}^{3+} \approx 0.03\right.$ at.\%); (ii) the $\mathrm{Er}^{3+} \mathrm{PL}$ is more efficient for samples with small size nanocrystallites embedded in an a-Si:H matrix; (iii) the PL quenching magnitude can not be related with the size of the Si crystals, but seems to be related with the presence of hydrogen; (iv) the $\mathrm{Er}^{3+} \mathrm{PL}$ is more temperature stable for the samples synthesized by Er co-sputtering than for the Er-implanted ones.

\section{Acknowledgment}

This work was partially supported by FCT Project POCTI/CTM/39395/2001 and INTAS Project \#03-51-6486.

\section{References}

[1] M. Fujii, M. Yoshida, Y. Kanzawa, S. Hayashi, K. Yamamoto, Appl. Phys. Lett. 71 (1997) 1198

[2] M. Fujii, M. Yoshida, S. Hayashi, K. Yamamoto, J. Appl. Phys. 84 (1998) 4525

[3] P.G. Kik, M. L. Brongersma, A. Polman, Appl. Phys. Lett 76 (2000) 2325

[4] M. Fujii, M. Yoshida, Y. Kanzawa, S. Hayashi, K. Yamamoto, Appl. Phys. Lett. 71 (1997) 1198

[5] G. Qin, G.G. Qin, S.H. Wanget., J. Appl. Phys. 85 (1999) 6738

[6] F. Priolo, G. Franzo, F. Iacona, D. Pacifici, V. Vinciguerra, Mat. Sc. Eng. B 81 (2001) 9

[7] P.G. Kik, A. Polman, Mat. Sc. Eng. B 81 (2001) 3

[8] M. Losurdo, M.M. Giangregorio, P. Capezzuto, G. Bruno, M.F. Cerqueira, E. Alves, M. 
Stepikhova, Appl. Phys. Lett. 83 (2002) 2993

[9] M.F. Cerqueira, M. Andritschky, L. Rebouta, J.A. Ferreira and M.F. Da Silva, Vacuum 46 (1995) 1385

[10] A.R. Zanatta and F.L. Freire Jr, Phys. Rev. B 62 (2000) 2016

[11] G.N. van Hoven, J.H. Shin, A. Polman, S. Lombardo and S.U. Campisano, J. Appl. Phys. 78 (1995) 2642

[12] R. Serna, E. Snoeks, G.N. van Hoven and A. Polman, J. Appl. Phys. 75 (1994) 2644

[13] M.F. Cerqueira, J.A. Ferreira, G. Adriaenssens, Thin Solid Films 370 (2000) 128

[14] D.A.G. Bruggemann, Ann. Phys. (Leipzig) 24 (1935) 636

[15] M. Losurdo, R. Rizzoli, C. Summonte, G. Cicala, P. Capezzuto, G. Bruno, J. Appl. Phys. 88 (2000) 2408

[16] P. Lautenschlager, M. Garriga, L. Vina, M. Cardona, Phys. Rev. B 36 (1987) 4821

[17] O.B. Gusev et al., Phys. Rev. B 64 (2001) 075302

[18] H. Przybylinska, W. Jantsch, Yu. Suprun-Belevitch, M. Stepikhova, L. Palmetshofer, G. Hendorfer, A. Kozanecki, R.Y. Wilson, B.J. Sealy, Phys. Rev. B 54 (1996) 2532 